\title{
Analysis of the asymmetric breakdown characteristics of trench isolation structure by using TCAD
}

\author{
S. Kumashiro, H. Kawaguchi, İ. Imai, and H. Matsumoto \\ ULSI Device Development Laboratories, NEC Corporation \\ 1120 Shimokuzawa, Sagamilıara, İanagawa 229-1198, JAPAN
}

\begin{abstract}
Experimentally observed asymmetric breakdown characteristics of shallow trench isolation are investigated by using TCAD. Both transient enlanced diffusion of boron and phosphorus and deactivation of phosphorus piled up at the trench bottom are the main causes of the asymmetry. Several techniques which reduce the asymmetry are proposed and their effects are compared by using TCAD.
\end{abstract}

\section{Introduction}

Shallow Trench Isolation (STI) is an indispensable technology for deep sub-micron ULSI. However, neither the dominant factors which determine the brealidown voltage of STI nor the proper guidelines for the well profile clesign are well understood. In this paper, experimentally observed asymmetric brealidown characteristics of STI are investigated and dominant factors for the breakdown characteristics of STI are clarified by using TCAD.

\section{Experiment and Simulation}

Two types of STI structure were fabricated as shown in Fig. 1. In the type-(a) structure, the well was formed after STI fabrication by ion implantation tilted by 7 elegrees to the n-well side. In contrast, the well ion implantation of the type-(b) structure was tilted to the p-well side. The conditions of the well ion implantation were phospliorus (P) $700 \mathrm{keV} 1.5 \mathrm{E} 13 \mathrm{~cm}^{-2}$ for the $n$-well and boron (B) $300 \mathrm{keV}$ $2 \mathrm{E} 13 \mathrm{~cm}^{-2}$ for the $\mathrm{p}$-well. The trench clepth was $550 \mathrm{~mm}$ and the width was varied from $200 \mathrm{~nm}$ to $1 \mu \mathrm{m}$. The breakdown characteristics due to the punch-through between the S/D region and the well on the opposite sides of the trench were measured by monitoriug the leak current. The measurement conclition is shown in Fig. 2. TCAD simulation was also performed for tliese STI structures. A process simulator which includes a crystal-mode Monte Carlo ion implantation model [1], a consistent pointdefect pair diffusion model [2], and a $P$ pile-11] moclel [3] and a drift-cliffusion type device simulator [4] were used. Fig. 3(a) shows the measured and the simulated leak current between the $n$-well and the $n+S / D$ region for the type-(a) structure. Neitlier 
the measured nor the simulated results show any sign of breakclown at the separation width (= half of the trench width) of $200 \mathrm{~nm}$. On the other hand, for the type-(b) structure, both the measured and the simulated results show significant breakdown between the $p$-well and the $p+S / D$ region at the separation width of $300 \mathrm{~nm}$ as shown in Fig. 3(b).

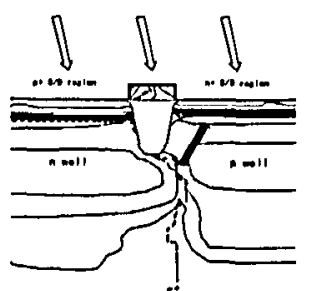

(a)

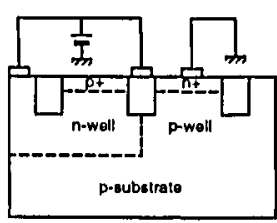

(a)

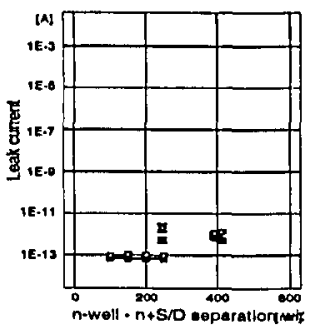

(a)

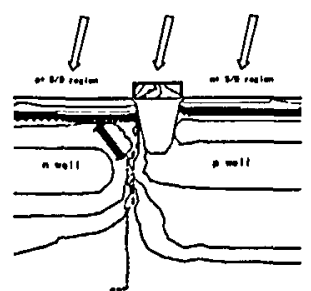

(b)

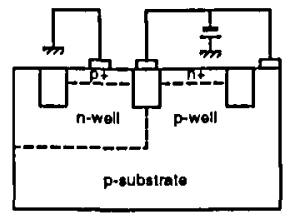

(b)

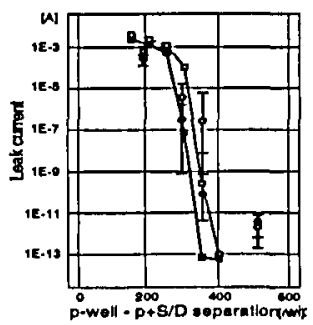

(b)

Fig. 1 Fabricated STI structures. (a) well $I / I$ is tilted to the n-well side. (b) well I/I is tilted to the p-well side. Weak patlis for brealidown are shown by the black arrows.

Fig. 2 Mensurement condition for the leak current. (a) between $n-w c l l$ and $n+S / D$ region. (b) between $\mathrm{p}$-well and $p+S / D$ region.

Fig. 3 Measured and simulated leak current for STI structures. (a) type-(a) structure. (b) type-(b) structure. Circles are measurcment and squares are simulation. Open symbols are for $4 \mathrm{~V}$ bias and closed symbols are for $2 \mathrm{~V}$ bias.

\section{Origin of the Asymmetry}

According to the experiment and the simulation, it was found that there was significant asymmetry in the breakelown characteristics between the type-(a) and the type-(b) structures, while their structures were symmetric. The implanted dose of the well was the only structural difference. However, according to simulation, it was found that strong asymmetry still remained even if the $P$ dose of the n-well was increased to $1 \mathrm{E} 13 \mathrm{~cm}^{-2}$. In order to clarify the origin of this asymmetry, a STI structure with the well formed by non-tilted and equally-closed ion implantation (i.e. completely symmetric structure) was simulated. Figs. 4-6 are the simulation results. Fig. 4(a) shows the as-implanted total $P$ and (b) shows the final active $P$ profiles of the n-well. It is observed that the final active concentration of $P$ around the bottom of the STI is smaller than the as-implanted concentration. Fig. 5(a) is the as-implanted total $B$ and (b) is the funal active $B$ profiles of the p-well. In contrast to $P$, the final B concentration around the bottom of the STI is larger than the initial one. Fig. 6 shows the variation of $P$ and $B$ concentration along the horizontal line near the 
bottom of the STI. At the time of ion implantation, the n-well and the p-well meet almost in the middle of the STI. However, after device fabrication, the p-well becomes dominant around the bottom of the STI and this causes asymmetric breakdown characteristics. The mechanism of the re-distribution of $B$ and $P$ is as follows: (1) Well ion implantation generates point-defects, which induce Transient Enhanced Diffusion (TED) of $B$ and $P$ during the subsequent thermal process. (2) As a result of TED, B and $P$ pile up at the trench-substrate interface which acts as a sink of point-defects. (3) The piled-up $P$ becomes inactive, while the piled-up $B$ remains active. Hence. B concentration becomes dominant around the bottom of the STI. In the conventional LOCOS process, an important factor for the well profile design is B dose loss from the substrate due to segregation during field oxidation. On the other hand, in the STI process, TED and pile up of dopant become important issues to be taken care of.

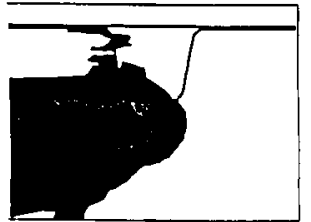

(a)

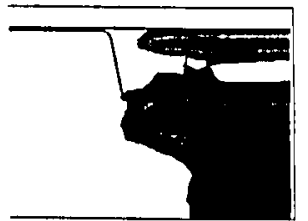

(a)

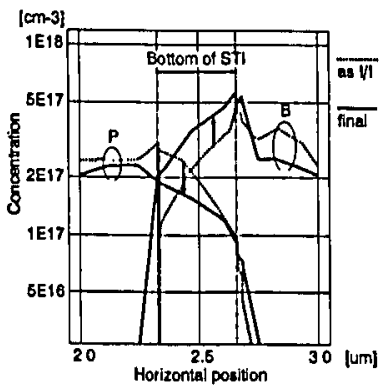

Fig. 4 Simulated P profile. (a) as implanted total concentration. (b) final active concentration. (b)

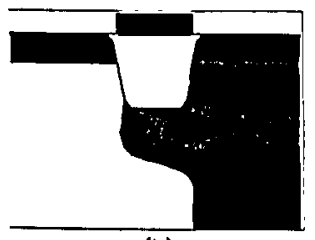

(b)

Fig. 5 Simulated B profile. (a) as implanted total concentration. (b) final active concentration.

Fig. $6 \mathrm{~B}$ and $\mathrm{P}$ concentration at the bottom of STI. Broken line: as implanted total concentration. Solidline: final active concentration. At the time of ion implantation, nwell and p-well meets almost in the middle of STI. After device fabrication, p-well becomes dominant at the bottom.

\section{Well Profile for Asymmetry Reduction}

In order to reduce the asymmetry in the breakdown characteristics, the following methods are proposed. (1) Increase the $P$ dose; that is, compensate the deactivated $P$ concentration. (2) Reduce the B implantation energy; that is, let B pile up at the side of the STI rather than at the bottom. (3) Reduce the P implantation energy; that is, set higl concentration region of $P$ between the $p+S / D$ region and the bottom of the STI. (4) Use As with the same projection range of $P$; that is, reduce TED and 
pile up effects. Effects of these methods were compared by using TCAD and the results are shown in Figs. 7-10. Method (3) is found to be the most effective.

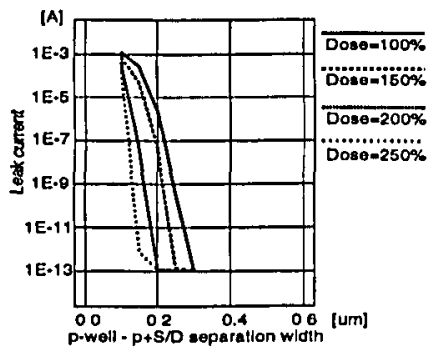

Fig. 7 Dependence of the leak current between $p$-well and $p+S / D$ region on P I/I dose.

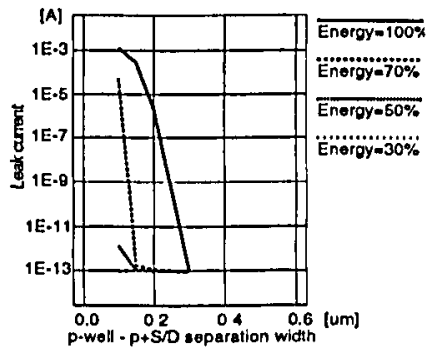

Fig. 9 Dependence of the leak current between $p$-well and $p+S / D$ region on P I/I encrgy.

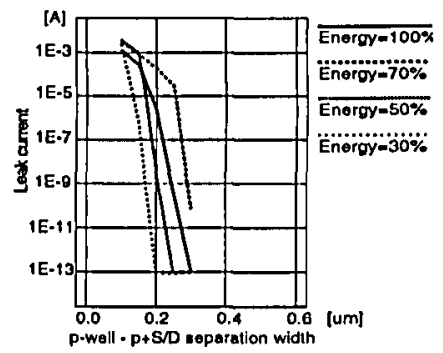

Fig. 8 Dependence of the leak current between $p$-well and $p+S / D$ region on B I/I energy.

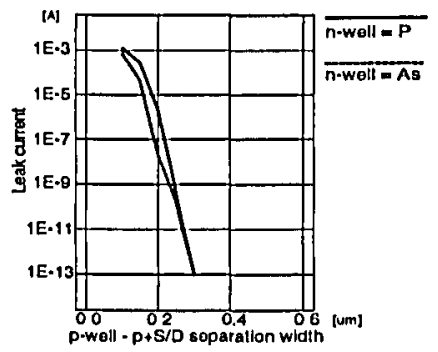

Fig. 10 Dependence of the leak current between $p$-well and $p+S / D$ region on I/I clopant.

\section{Conclusion}

It is shown that both TED of $B$ and $P$ and deactivation of $P$ piled up at the trench bottom are the main causes of the asymmetric brealiclown characteristics of the STI structure. Reduction of the P implantation energy is found to be effective in reducing the asymmetry.

\section{References}

[1] M. Hane and M. Fukuma, "Ion implantation model considering crystal structure effects," IEEE Trans. Electron Devices, vol. 37, no. 9, pp. 1959-1063, 1990.

[2] H. Sakamoto, S. Kiumashiro, M. Hiroi, M. Hane, and H. Matsumoto, "Simulation of reverse short channel effects with a consistent point-clefect diffusion model," SISPAD'97, p]. 137-140, 1997.

[3] H. Sakamoto and S. Kumashiro, "A new diffusion algorithm duriug oxidation which can handle both phosphorus pile-up and boron segregation at $\mathrm{Si}_{-} \mathrm{SiO}_{2}$ interface," SISPAD'07, pp. 81-84, 1997.

[4] S. Kumashiro and M. Sakurai, "Two-dimensional two-carricr gencral purpose device simulator BIUNAP," NASECODE IV, pp. 365-371, 1985. 